$05,13,01$

\title{
Изучение процесса перемагничивания пермаллоевых микрочастиц с конфигурационной анизотропией методами магнитно-силовой микроскопии
}

\author{
(C) Д.А. Бизяев ${ }^{1}$, А.А. Бухараев ${ }^{1,2}$, А.П. Чукланов ${ }^{1}$, Н.И. Нургазизов ${ }^{1}$ \\ ${ }^{1}$ Казанский фризико-технический институт им. Е.К. Завойского ФИЦ КазНЦ РАН, \\ Казань, Россия \\ ${ }^{2}$ Казанский (Приволжский) федеральный университет, \\ Казань, Россия \\ E-mail: dbiziaev@inbox.ru
}

\begin{abstract}
Методами магнитно-силовой микроскопии проведено исследование распределения намагниченности в микрочастицах пермаллоя, обладающих конфигурационной анизотропией. Изучались треугольные частицы с различной степенью прогиба боковых сторон. Анализ полученных данных позволил установить, что частицы могут находиться в нескольких квазиоднородных стабильных состояниях. Было показано, что в зависимости от ориентации частицы относительно внешнего магнитного поля ее перемагничивание может происходить как скачкообразно, так и через промежуточное состояние. Продемонстрировано, что ориентацию квазиоднородной намагниченности в частице можно менять зондом магнитно-силового микроскопа.
\end{abstract}

DOI: 10.21883/FTT.2018.11.46656.06NN

\section{1. Введение}

Плоские ферромагнитные частицы представляют интерес в качестве паттернированной среды для сверхплотной магнитной записи информации. Традиционно такие частицы имеют классическую форму (эллиптическую или прямоугольную) и являются однодоменными. Для изменения направления их намагниченности обычно используется внешнее магнитное поле. Однако величина поля необходимого для переключения между двумя стабильными состояниями в таких частицах достаточно большая [1,2]. Для уменьшения величины этого поля часто дополнительно используют различные внешние факторы, такие как температура или механические напряжения [2-4].

Уменьшить величину поля переключения частицы, сохранив при этом стабильность ее состояния можно за счет использования частиц, обладающих конфигурационной анизотропией формы [5-8], например треугольных или многолепестковых. Такие частицы, за счет более сложной формы, могут находиться в нескольких квазиоднородных стабильных состояниях и таким образом хранить не один, а несколько бит информации. Изучение процессов переключения таких частиц из одного магнитного состояния в другое вызывает определенный научный интерес.

В представленной работе при помощи магнитно-силового микроскопа (МСM) изучались процессы перемагничивания трех типов частиц пермаллоя (Ру), имеющих конфигурационную анизотропию. Исследовались планарные частицы треугольной формы, которые отличались различной степенью прогиба боковых сторон. Проводилось перемагничивание всего сформированного массива частиц при помощи внешнего магнитного поля и индивидуальной частицы при помощи МСМ-зонда.

\section{2. Методики приготовления образцов и проведения измерений}

Исследованные в работе Ру-частицы были созданы при помощи сканирующей зондовой литографии (СЗЛ) и методики lift-off [9]. Полученные частицы были расположены на поверхности кремниевой подложки. В качестве материала для формирования защитной маски использовался полиметилметакрилат (ПММА). Полимерная маска на поверхности подложки формировалась в пленке толщиной $100 \mathrm{~nm}$, равномерность которой достигалась за счет высыхания капли раствора ПММА в хлороформе $(0.55 \%)$ при непрерывном вращении подложки (так называемая методика spin-coating). Для СЗЛ использовался кантилевер D300 (SCDprobes) c алмазным пирамидальным монокристаллом, закрепленным на кремниевой балке и сканирующий зондовый микроскоп (C3M) Solver P47Pro, работающий в режиме атомно-силового микроскопа (АСМ). На площади размером $50 \times 50 \mu \mathrm{m}^{2}$ формировался массив частиц и специальная метка, при помощи которой можно было в дальнейшем легко найти полученные частицы на поверхности подложки, контролируя положение кантилевера СЗМ с помощью оптического микроскопа. Размер используемой подложки составлял $15 \times 3 \mathrm{~mm}^{2}$, толщина $0.4 \mathrm{~mm}$. В качестве подложки использовался полированный низкоомный кремний, с шероховатостью поверхности $3 \mathrm{~nm}$.

Для нанесения слоя пермаллоя (сплав 79НМ, основные компоненты: $\mathrm{Ni} 79 \%$, Fe 16\%, Мо 4\%) использовалась методика распыления электронным лучом твердотельной мишени. Напыление проводилось в условиях сверхвысокого вакуума на установке Omicron Multiprobe P. Далее излишки металла и ПММА удалялись в ультразвуковой ванне при помощи хлороформа. 

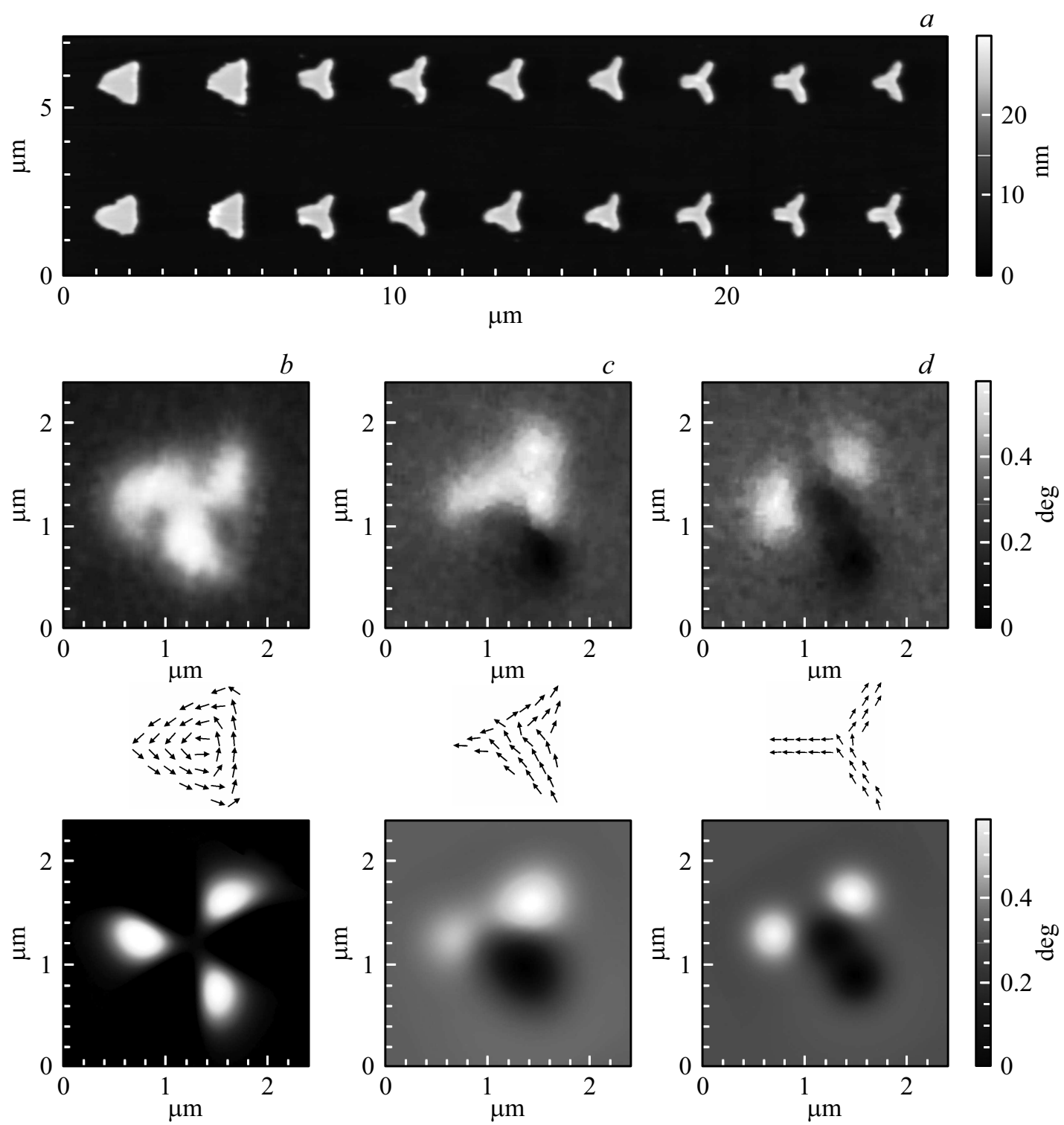

Рис. 1. АСМ-изображение части массива Ру-частиц, сформированных на кремниевой подложке $(a)$. МСМ-изображение отдельной частицы первого $(b)$, второго $(c)$ и третьего $(d)$ типов. Под каждым изображением соответствующее ему распределение локальной намагниченности в частице, смоделированное при помощи ООММF и виртуальное МСМ-изображение, полученное на основе данного распределения намагниченности.

Затем образец промывался дистиллированной водой и высушивался. Сформированный массив состоял из трех типов частиц, которые отличались друг от друга формой и имели одинаковую высоту равную $30 \mathrm{~nm}$ (рис. 1,a). Первый тип представлял собой частицы, которые в плоскости имели форму равнобедренного треугольника со стороной равной $1.35 \mu \mathrm{m}$ (рис. $1, b)$. У второго типа частиц также было три угла, однако они имели вогнутые к центру криволинейные стороны (рис. 1,c). Длина стороны треугольника, в который можно было вписать такую частицу, также составляла $1.35 \mu \mathrm{m}$. Частицы третьего типа были сформированы в виде трехлучевой звезды и имели форму похожую на букву „,““ (рис. 1,d). Они также могли быть вписаны в треугольник со стороной $1.35 \mu \mathrm{m}$, при этом ширина выступающих частей составляла $400 \mathrm{~nm}$. Будем в дальнейшем также называть частицы третьего типа Ү-частицами.

Распределение намагниченности в полученных частицах исследовалось с помощью С3М Solver P47Pro. Для МСМ-измерений использовались стандартные коммерческие кантилеверы NSC 18/Co-Cr (MikroScience). Во время МСМ-измерений использовалась однопроходная методика, при которой МСМ-зонд перемещается над поверхностью образца на достаточном удалении (несколько десятков нанометров) для того, чтобы исключить перемагничивание частиц магнитным полем зонда. Для проведения МСМ-измерений во внешнем магнитном поле в СЗМ был установлен электромагнит, позволяющий изменять напряженность внешнего магнитного поля в плоскости образца в интервале от 


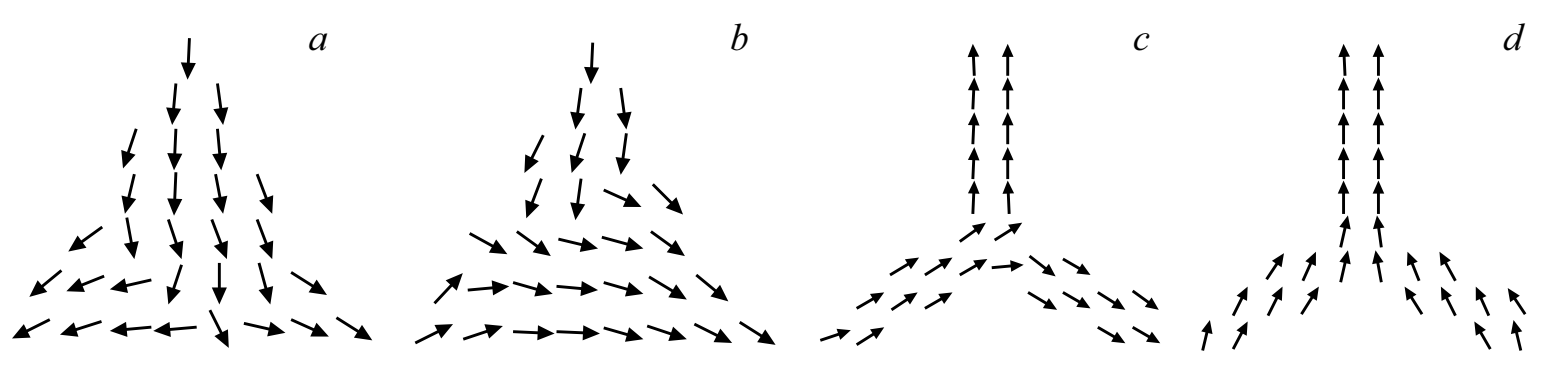

Pис. 2. Два типа локального распределения намагниченности в частицах второго $(a, b)$ и третьего $(c, d)$ типа, смоделированные в программе OOMMF.

$-16.5 \mathrm{mT}$ до $+16.5 \mathrm{mT}$ с шагом $0.5 \mathrm{mT}$. Протяжка поля осуществлялась от положительных значений к отрицательным. Перед проведением измерений частицы были намагничены во внешнем поле величиной $+200 \mathrm{mT}$.

Для определения распределения намагниченности в частице, исходя из полученного МСМ-изображения, использовался следующий подход. Возможное распределение намагниченности в частице моделировалось при помощи программы OOMMF [10]. При этом использовалось ее трехмерное изображение, полученное при АCМ-измерениях. Затем по рассчитанному распределению намагниченности моделировалось виртуальное МСМ-изображение частицы при помощи разработанной ранее программы „Виртуальный микроскоп“ [11]. Полученное изображение сравнивалось с экспериментальным и при их совпадении делался вывод о том, что полученное распределение намагниченности соответствует реальному. При моделировании частица разбивалась на элементарные кубические ячейки с размером грани $4 \mathrm{~nm}$. Считалось, что намагниченность насыщения пермаллоя равна $750 \mathrm{kA} / \mathrm{m}[12]$. В связи с тем, что исследуемые частицы имели поликристаллическую структуру, константа магнитной кристаллической анизотропии принималась равной нулю.

\section{3. Магнитно-силовая микроскопия пермаллоевых частиц с конфигурационной анизотропией}

При проведении МСМ-исследований частиц первого типа (треугольной формы), было установлено, что в свободном состоянии (при отсутствии механических напряжений и внешнего магнитного поля) они обладают вихревой структурой намагниченности (рис. $1, b)$. У частиц второго типа (треугольные частицы с вогнутыми внутрь сторонами) в свободном состоянии наблюдается квазиоднородная намагниченность (рис. 1,c). Учитывая симметрию частицы, она может находиться в одном из шести состояний с квазиоднородной намагниченностью. Два основных варианта распределения локальной намагниченности в частице представлены на рис. $2, a, b$. Еще четыре варианта намагниченности получаются путем поворота каждого из представленных распределений на $120^{\circ}$ и $240^{\circ}$. Во всех случаях общее направление намагниченности частицы совпадает с одной из медиан треугольника, в который может быть вписана частица. Данное направление является осью легкого намагничивания (ОЛН) частицы, что совпадает с результатами исследований других авторов [6]. Аналогично [6], направление параллельное одной из сторон треугольника будет осью трудного намагничивания (ОТН) в плоскости частицы.

У частиц третьего типа (Ү-частиц) в свободном состоянии также наблюдается квазиоднородная намагниченность (рис. $1, d)$. При этом получаемые МСМ-изображения несколько отличаются от МСМ-изображений частиц второго типа. В упрощенном варианте можно считать, что намагниченность каждого из трех лучей может быть направлена к центру или от центра частицы. Варианты, когда намагниченность всех лучей имеет одинаковое направление к центру или от центра частицы энергетически не выгодны, поэтому Ү-частицы могут находиться в одном из шести состояний квазиоднородной намагниченности, которым будут соответствовать три ОЛН. Два возможных варианта намагниченности Ү-частицы представлены на рис. $2, c, d$ еще четыре варианта получаются при повороте представленных распределений на угол $120^{\circ}$ и $240^{\circ}$ по часовой стрелке.

Перемагничивание частиц внешним магнитным полем изучалось при двух ориентациях поля относительно частиц, исходя из соображений о симметрии магнитных состояний частицы. Магнитное поле прикладывалось в направлениях вдоль одной из медиан и вдоль одной из сторон треугольника, в который могла быть вписана частица. Согласно полученным данным о распределении намагниченности эти направления соответствуют одной из ОЛН и ОТН для частиц второго и третьего типа. Перед началом экспериментов все частицы намагничивались в одну сторону во внешнем магнитном поле напряженностью $+200 \mathrm{mT}$.

Проведенные измерения показали, что при уменьшении положительного значения внешнего магнитного поля, приложенного вдоль медианы треугольника (параллельно ОЛН), однородная намагниченность частиц первого типа сохранялась вплоть до значения $+4 \mathrm{mT}$. Затем частицы начинали переходить в состояние с вихревой намагниченностью (рис. 1). Все исследуемые частицы оказывались в состоянии с вихревой намагниченностью при величине поля $+2 \mathrm{mT}$. Наблюдаемый 
процесс перемагничивания треугольных частиц существенно отличается от изменения магнитной структуры эллиптических Ру-частиц во внешнем магнитном поле (высота $30 \mathrm{~nm}$, длина вдоль короткой оси $200 \mathrm{~nm}$, аспектное соотношение 4:1 и менее) исследованного в работе [13]. В таких эллиптических частицах процесс перехода в вихревое состояние начинался только после смены направления внешнего магнитного поля на противоположное. Однородное намагничивание частиц первого типа в противоположном направлении начиналось при значении поля $-7 \mathrm{mT}$. При поле $-8 \mathrm{mT}$ все частицы становились однородно намагниченными в противоположном направлении. Для эллиптических частиц с аспектным соотношением меньше чем 4:1, в которых процесс перемагничивания происходил через образование вихревого состояния, однородное намагничивание происходило в полях $-15 \mathrm{mT}$ [13], что существенно больше чем для треугольных частиц. При ориентации внешнего магнитного поля вдоль стороны треугольника (параллельно ОТН) переход частиц в вихревое состояние происходил при магнитном поле в диапазоне $+3-+1.5 \mathrm{mT}$, а однородное намагничивание в противоположном направлении при полях $-8--9 \mathrm{mT}$.

Для частиц второго типа, при внешнем магнитном поле, приложенном вдоль медианы треугольника (или параллельно направлению квазиоднородной намагниченности частицы) практически во всех случаях перемагничивание частицы происходило скачкообразно при значениях поля $-4--8 \mathrm{mT}$. Какого-либо промежуточного состояния на полученных МСМ-изображениях не наблюдалось, и частица сохраняла свою квазиоднородную намагниченность. С частицами второго типа можно сравнить эллиптические частицы с аспектным соотношением больше чем 4:1, в которых процесс перемагничивания вдоль ОЛН также происходил скачкообразно во внешних магнитных полях от -7 до $-12 \mathrm{mT}$ [13]. Эти значения превосходят величину поля перемагничивания, наблюдаемую для частиц второго типа. При ориентации внешнего магнитного поля вдоль стороны треугольника (параллельно ОТН) переключение частицы в состояние с противоположным направлением намагниченности происходило практически во всех случаях скачкообразно при значениях поля $-2.5--9 \mathrm{mT}$ (рис. 3). Только у одной частицы намагниченность менялась через состояние, когда направление квазиоднородной намагниченности было перпендикулярно внешнему полю (рис. 3,c). Для остальных частиц промежуточные состояния на полученных МСМ-изображениях не наблюдались, и частицы сохраняли направление квазиоднородной намагниченности до момента переключения.

Для частиц третьего типа при внешнем магнитном поле, приложенном вдоль медианы треугольника (параллельно ОЛН), перемагничивание частицы также происходило скачкообразно при значениях поля $-5.5--11 \mathrm{mT}$, без промежуточных состояний. Наблюдаемые значения полей для Ү-частиц становятся сопоставимыми с полями переключения частиц эллиптической формы с аспектным соотношением сторон 7:1
(-7--12 mT [13]), что связано с большой анизотропией формы Ү-частиц по сравнению с треугольными частицами первых двух типов.

При ориентации внешнего магнитного поля вдоль стороны треугольника (или вдоль ОТН) поведение частицы в магнитном поле менялось. Здесь довольно часто переключение частицы происходило через промежуточное квазиоднородное состояние (рис. 3,e). При этом данное направление намагниченности частицы было повернуто на угол $90^{\circ}$ к направлению внешнего магнитного поля. Переход в промежуточное состояние начинался при значении поля $-2.5 \mathrm{mT}$ (рис. $3, c$ ). Основная масса частиц переходила в промежуточное квазиоднородное состояние при значениях поля $-8--9 \mathrm{mT}$ (рис. 3,e). Переход частиц в состояние с противоположным начальному направлению намагниченности начинался при значении внешнего магнитного поля $-8 \mathrm{mT}$ и при поле $-16 \mathrm{mT}$ все частицы оказывались в этом состоянии (рис. $3, f)$. Процесс переключения частицы внешним магнитным полем был смоделирован в программе ООММF, и было установлено, что направление квазиоднородной намагниченности изменяется на противоположное при значении внешнего поля $11 \mathrm{mT}$.

Наблюдаемый разброс поля переключения Ү-частиц $(-8--15 \mathrm{mT})$, на наш взгляд, связан с разной формой получаемых частиц и наличием у них дефектов, что обусловлено методикой создания. Наличие промежуточного квазиоднородного состояния в процессе перемагничивания Ү-частицы в направлении параллельном стороне треугольника, в который можно вписать частицу, подтверждает заключение о том, что данное направление является ОТН частицы. Отсутствие промежуточного состояния при ориентации внешнего поля параллельно медиане треугольника свидетельствует о том, что данное направление является ОЛН частицы.

Магнитная структура треугольных Ру-частиц со стороной $1.35 \mu \mathrm{m}$ исследованных в данной работе заметно отличается от структуры аналогичных частиц с размером $0.5 \mu \mathrm{m}$, рассмотренных ранее в работе [6]. Треугольные частицы с субмикронными размерами [6] имеют ОЛН вдоль сторон треугольника, а ОТН вдоль медиан. При этом прогиб сторон треугольной субмикронной частицы приводит к тому, что ОЛН и ОТН меняются местами. В нашем случае рассматриваемые частицы имеют микронные размеры и, согласно полученным МСМ-данным, ОЛН всех частиц направлено вдоль медианы треугольника, а ОТН вдоль его стороны. Это свидетельствует о том, что в данном случае основным фактором, влияющим на намагниченность частицы, является анизотропия формы, а не ее размер. При этом увеличение прогиба ведет к увеличению поля анизотропии частицы. Из этого следует, что для частиц одинаковой толщины, как в нашем случае, поле анизотропии частицы обратно пропорционально площади частицы. Это в свою очередь сказывается на величине энергии потенциального барьера между двумя квазиоднородными состояниями намагниченности, которая 

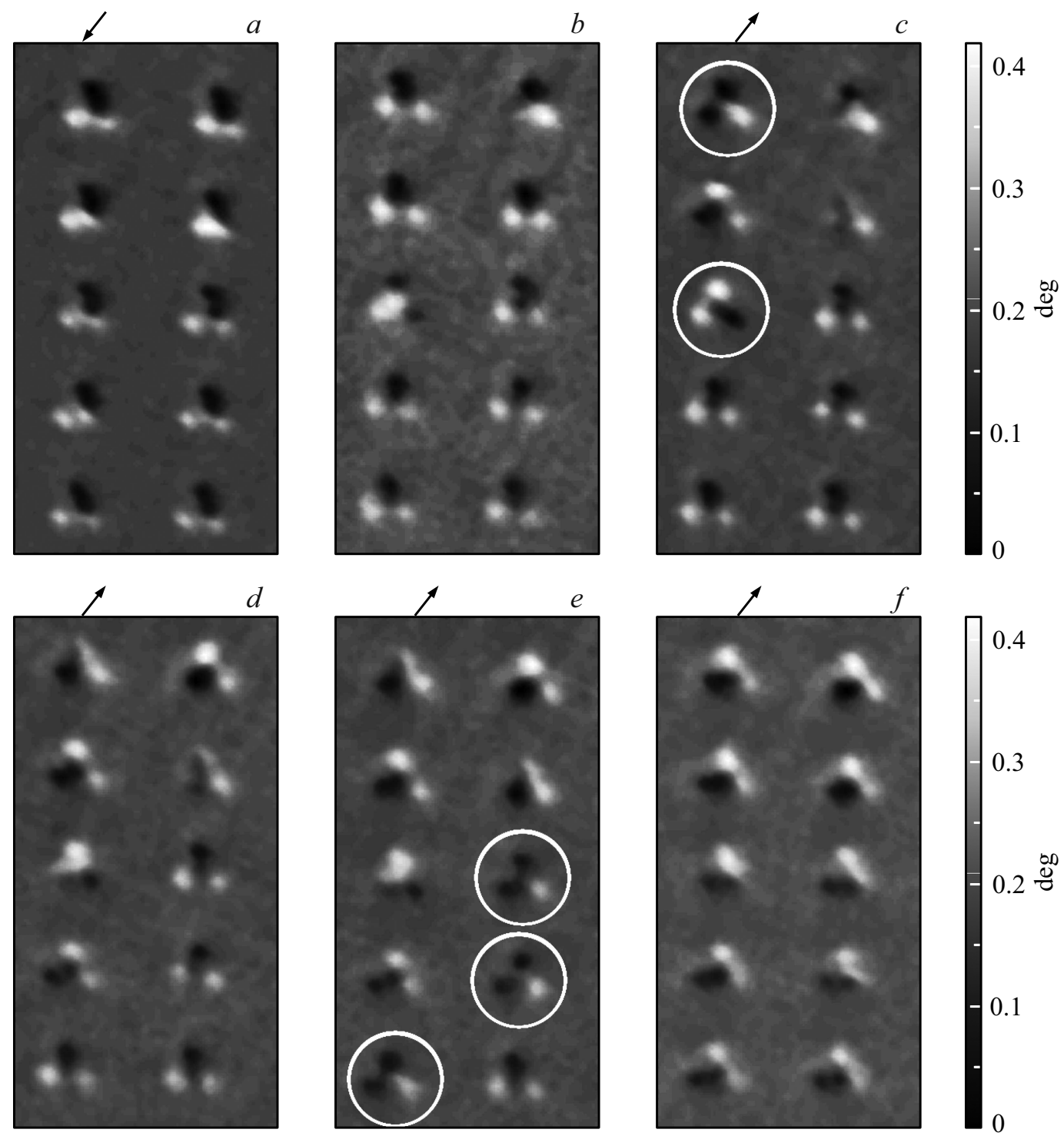

Рис. 3. МСМ изображение частиц второго (верхние 2 ряда) и третьего (нижние три ряда) типа находящихся во внешнем магнитном поле напряженностью: $+16 \mathrm{mT}-(a), 0 \mathrm{mT}-(b),-2.5 \mathrm{mT}-(c),-5.5 \mathrm{mT}-(d),-9 \mathrm{mT}-(e),-16 \mathrm{mT}-(f)$. Направление поля указано стрелкой сверху. Размер скана $14.4 \times 7.5 \mu \mathrm{m}^{2}$. Кругами отмечены частицы, квазиоднородное направление намагниченности которых перпендикулярно внешнему полю.
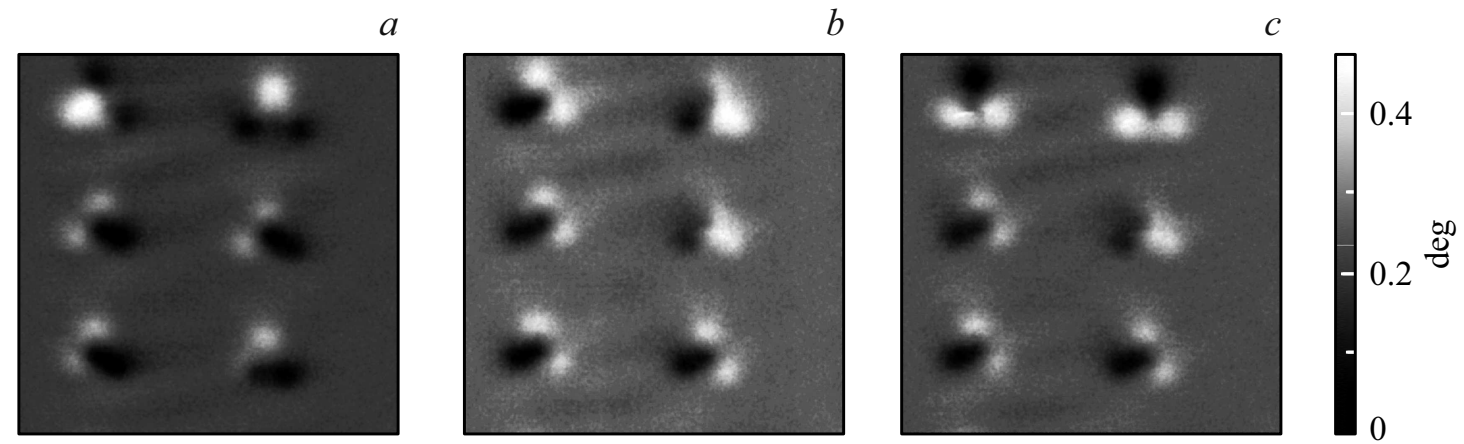

Рис. 4. Перемагничивание Ү-частиц МСМ-зондом. МСМ-изображения исходного магнитного состояния частиц $(a)$, изменение состояния всех шести частиц, за счет сканирования их всех МСМ-зондом в контакте $(b)$, и изменение состояния только двух верхних частиц, после сканирования их в контакте (c). Размер скана $9 \times 9 \mu \mathrm{m}^{2}$. 
прямо пропорциональна энергии анизотропии. По этой причине, перемагничивание микронных частиц второго типа, наблюдаемое при МСМ-измерениях, почти во всех случаях происходило скачкообразно (без промежуточных состояний) независимо от ориентации внешнего поля к ОЛН, поскольку их площадь больше, чем у частиц третьего типа.

Промежуточные состояния, наблюдаемые у Ү-частиц при их перемагничивании магнитным полем, направленным вдоль ОТН, обусловлены тем, что в этом случае метастабильные состояния намагниченности частицы находятся под углом $90^{\circ}$ к внешнему полю. При этом полная энергия частицы во внешнем магнитном поле снизится за счет того, что энергия Зеемана будет равна нулю, в отличие от других ориентаций: $E_{z}=-M \cdot H \cdot \cos \theta$, где $M-$ намагниченность частицы, $H-$ внешнее магнитное поле, $\theta-$ угол между направлением намагниченности и внешним полем. Это приводит к тому, что частица в таком состоянии оказывается более стабильной в отличие от других ориентаций относительно внешнего поля.

Полученные при экспериментах значения магнитного поля необходимого для перемагничивания частиц второго и третьего типа сопоставимы по своей величине с магнитным полем создаваемым МСМ-зондом, который можно использовать для индивидуального изменения направления намагниченности частицы или гетероструктуры [14]. Перед проведением процесса перемагничивания частиц с помощью МСМ-зонда подбиралась оптимальная высота сканирования образца по однопроходной методике, при которой не происходило перемагничивания частиц, и фиксировалось их начальное состояние (рис. 4, a). Затем МСМ-зонд приводился в контакт с поверхностью, и производилось повторное сканирование. После этого зонд отводился от поверхности образца, и сканировалось полученное состояние частиц (рис. $4, b$ ). Таким образом, за один скан можно было менять направление намагниченности одной или нескольких частиц (рис. 4). Направление намагниченности частицы после такого сканирования зависело от направления перемещения МСМ-зонда и начального состояния частицы. У частиц первого типа, которые при отсутствии внешнего магнитного поля имеют вихревое состояние намагниченности, после воздействия на них с помощью МСМ-зонда, сканировавшего частицы в контактном режиме, в некоторых случаях наблюдалось изменение направления вихря. Таким образом, было показано, что с помощью МСМ-зонда можно было индивидуально менять направление намагниченности частицы.

\section{4. Заключение}

Проведенные исследования треугольных Ру частиц с различной степенью прогиба боковых сторон показали, что прогиб сторон треугольника приводит к появлению в частице квазиоднородного состояния намагниченности. Увеличение степени прогиба сторон частицы приводит к увеличению поля переключения частицы и образованию выраженных осей легкого и трудного намагничивания, что наблюдается на $Y$-частицах, которые имеют максимальный прогиб стенок. Перемагничивание таких частиц путем приложения внешнего магнитного поля, направленного параллельно ОТН, обычно происходит через промежуточное состояние с квазиоднородной намагниченностью, направление которого перпендикулярно внешнему магнитному полю.

\section{Список литературы}

[1] R.P. Cowborn. J. Phys. D 33, R1-R16 (2000).

[2] Н.И. Нургазизов, Т.Ф. Ханипов, Д.А. Бизяев, А.А. Бухараев, А.П. Чукланов. ФТТ 56, 1756 (2014).

[3] M.H. Kryder, E.C. Gage, T.W. McDaniel, W.A. Challener, R.E. Rottmayer, G. Ju, Y.-T. Hsia, M.F. Erden. Proc. IEEE 96, 1810 (2008).

[4] Y. Liu, Q. Zhan, B. Wang, H. Li, Y. Wu, B. Chen, D. Sun, S. Mao, R.-W. Li. IEEE Transact. Magn. 51, 2501404 (2015).

[5] M. Salehi-Fashami, N. D’Souza. JMMM 438, 76 (2017).

[6] K. Nanayakkara, I.S. Vasil'evskii, I.S. Eremin, O.S. Kolentsova, N.I. Kargin, A. Anferov, A. Kozhanov. J. Appl. Phys. 119, 233906 (2016).

[7] B. Lambson, Z. Gu, M. Monroe, S. Dhuey, A. Scholl, J. Bokor. Appl. Phys. A 111, 413 (2013).

[8] S. Giordano, Y. Dusch, N. Tiercelin, P. Pernod, V. Preobrazhensky. Phys. Rev. B 85, 155321 (2012).

[9] А.А. Бухараев, Д.А. Бизяев, Н.И. Нургазизов, Т.Ф. Ханипов. Микроэлектроника 41, 90 (2012).

[10] M.J. Donahue, D.G. Porter. OOMMF (http://math.nist.gov/oommf/).

[11] Д.В. Овчинников, А.А. Бухараев. ЖТФ 71, 85 (2001).

[12] И.К. Кикоин. Справочник. Атомиздат, М. (1976). 1008 с.

[13] X. Zhu, P. Grütter, V. Metlushko, B. Ilic. Phys. Rev. B 66, 024423 (2002).

[14] В.Л. Миронов, А.А. Фраерман, Б.А. Грибков, О.Л. Ермолаева, С.А. Гусев, С.Н. Вдовичев. ФТТ 52, 2153 (2010).

Редактор К.В. Емцев 